\title{
Sparklan Antenna in Indoor Positioning Application
}

\author{
Huang-Chu Huang, ${ }^{1}$ Chi-Yen Shen, ${ }^{2}$ Chih-Yung Chen, ${ }^{3}$ \\ Yu-Ju Chen, ${ }^{4}$ and Rey-Chue Hwang ${ }^{2 *}$ \\ ${ }^{1}$ Department of Electronic Communication Engineering, National Kaohsiung University of Science and Technology, \\ No. 142, Haijhuan Rd., Nanzih District., Kaohsiung City 81157, Taiwan, R.O.C. \\ ${ }^{2}$ Department of Electrical Engineering, I-Shou University, \\ No. 1, Sec. 1, Syuecheng Rd., Dashu District, Kaohsiung City 84001,Taiwan, R.O.C. \\ ${ }^{3}$ Department of Computer Science and Information Engineering, Shu-Te University, \\ No. 59, Hengshan Rd., Yanchao District, Kaohsiung City 82445, Taiwan, R.O.C. \\ ${ }^{4}$ Department of Information Management, Cheng-Shiu University, \\ No. 840, Chengcing Rd., Niaosong District, Kaohsiung City 83347, Taiwan, R.O.C.
}

(Received November 28, 2018; accepted July 10, 2019)

Keywords: Sparklan antenna, indoor positioning

In this paper, the study of a Sparklan antenna used for indoor positioning (IP) is presented. The completed sensing hardware infrastructure is composed of six Sparklan AX-106M antennas and one Zigbee module. An indoor area with a size of $64 \mathrm{~m}^{2}$ in the building of SueTe University is used as the experimental field and a database including 704 sensed points with six received signal strength (RSS) signals is firstly constructed as the reference object locations. The RSSs of six antennas are used for object positioning estimation using a fuzzy estimator. To demonstrate the superiority of the developed IP system (IPS), several experiments were conducted. Experimental results show that the positioning performance of the IPS we developed is still accurate even when some antennas are disabled.

\section{Introduction}

Currently, how to accurately locate the position of people or objects in a huge indoor space has become an important issue and some survey reports have also been presented. ${ }^{(1-3)}$ Thus, different indoor positioning (IP) systems constructed using different wireless sensing and communication elements have been developed and studied. ${ }^{(4-17)}$ For instance, in large shopping malls, airports or hospitals, a good IP system (IPS) could be used for the monitoring of people's movements. In a big factory, an IPS could be used for the positioning management of objects and equipment. A good functional IPS could also be used as a rescue system that can accurately provide the victims' position so that the rescue team can easily find the people who need help.

However, owing to the different components used, different IPSs have their advantages and disadvantages. Sparklan AX-106M is an antenna that can be combined with other wireless devices to perform the IP work. The descriptions of its function are shown in Table $1 .^{(18)}$ In this paper, we present an IPS that is composed of six Sparklan AX-106M antennas [denoted by

*Corresponding author: e-mail: rchwang@isu.edu.tw https://doi.org/10.18494/SAM.2019.2207 
Table 1

Functions of Sparklan AX-106M.

\begin{tabular}{lc}
\hline Frequency band & $2.4-2.5 \mathrm{GHz}$ \\
Antenna gain & $6 \mathrm{dBi}$ \\
VSWR & $1.5 \mathrm{max}$ \\
Polarization & Linear vertical \\
HPBW/H-plane & $80+-$ \\
HPBW/E-plane & $80+-$ \\
Impedance & $50 \Omega$ \\
Dimensions $H \times W \times D$ & $115 \times 76 \times 33 \mathrm{~mm}^{3}$ \\
Weight & $110 \mathrm{~g}$ without joint \\
\hline
\end{tabular}

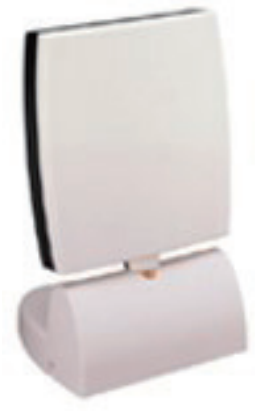

(a)

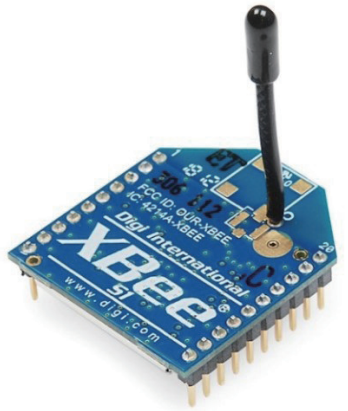

(b)

Fig. 1. (Color online) Pictures of (a) antenna and (b) Zigbee sensor.

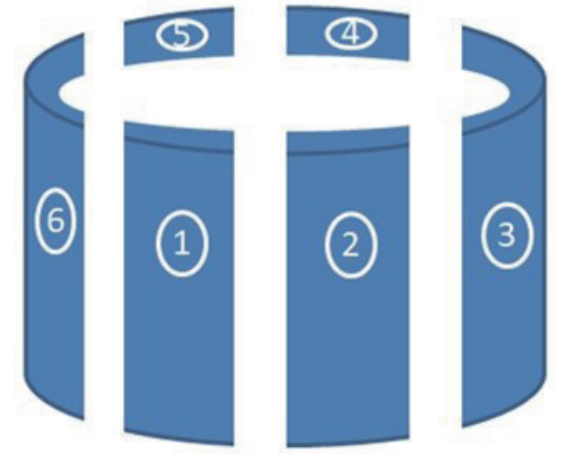

Fig. 2. (Color online) Schematic diagram of Sparklan antenna module.

numbers (1), (2), (3), (4), (5), and(6)] and one Zigbee module. The pictures of the antenna and Zigbee sensor are shown in Fig. 1 and the schematic diagram of the Sparklan antenna module is shown in Fig. 2.

A complete IPS must have the positioning estimation algorithm to calculate the coordinates of the object location. Thus, triangulation, scene analysis, and proximity are the three main estimation algorithms used in IP applications. Each algorithm has its specific function and limit. In this research, a fuzzy estimator is used to perform the object location estimation. The fuzzy estimation algorithm will be described in the next section.

To perform the IP experiments, a reference database that includes 704 sensed points is built firstly. Each sensed point has six received signal strength (RSS) signals and the coordinates of one object $\left\{\left[\left(x_{i}, y_{i}\right),\left(s_{1 i}, s_{2 i}, s_{3 i}, s_{4 i}, s_{5 i}, s_{6 i}\right)\right], i=1,2, \ldots, 704\right\}$. The diagram of 704 sensed points is shown in Fig. 3.

This paper is organized as follows. In Sect. 2, the fuzzy estimation algorithm is described. All experiments are presented in Sect. 3 and a conclusion is given in Sect. 4.

\section{IPS Fuzzy Estimator}

Over the past decades, fuzzy theory has been widely applied in many engineering and social science applications. ${ }^{(19)}$ It was initialized by Professor Lotfi Zadeh in 1965 . Usually, a fuzzy 


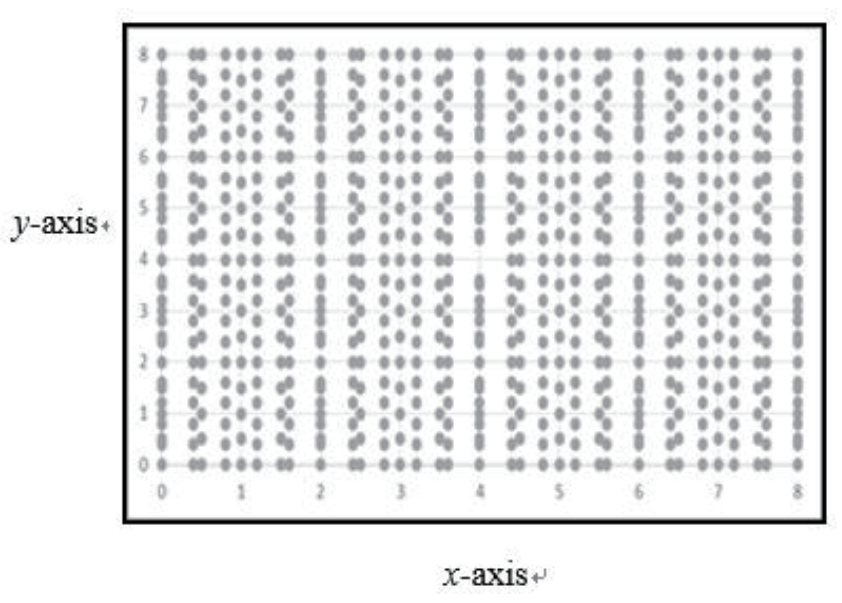

Fig. 3. Diagram of 704 points.

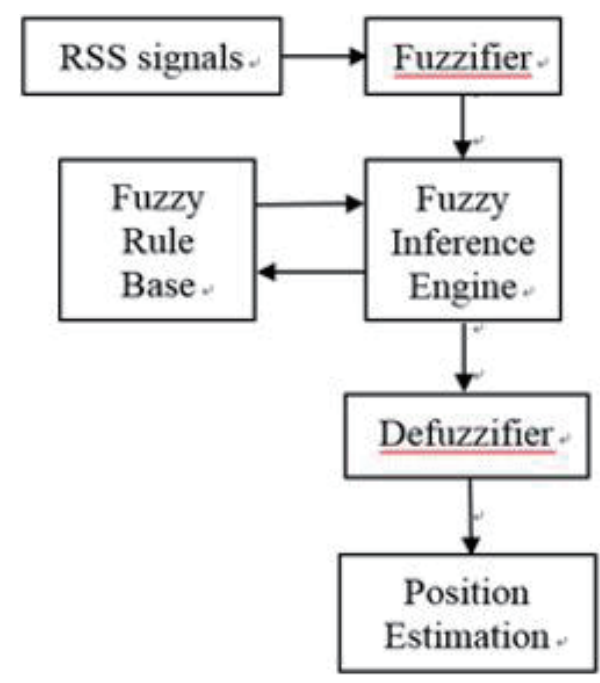

Fig. 4. IPS fuzzy estimator.

system is called a knowledge- or rule-based system, because it is a database consisting of many fuzzy IF-THEN rules, which are stated in accordance with the knowledge of experts. The fuzzy system used in this research is mainly constructed using a fuzzifier, a fuzzy rule base, a fuzzy inference engine, and a defuzzifier. Figure 4 shows the fuzzy estimator developed for our IPS. The detailed fuzzy estimation process is presented as follows. ${ }^{(20,21)}$

Now, we assume that the RSS signals sensed at a blind node $\left(x_{b}, y_{b}\right)$ are $\left(r_{1 b}, r_{2 b}, r_{3 b}, r_{4 b}\right.$, $\left.r_{5 b}, r_{6 b}\right)$ and that the fuzzy rule is constructed using the $m$ fuzzy IF-THEN rules expressed as follows.

Rule $^{(m-\text { th)})}$ IF $r_{1 b}$ is $s_{1 m}$ and $r_{2 b}$ is $s_{2 m}$ and $\ldots$ and $r_{6 b}$ is $s_{6 m}$, THEN $\left(x_{b}=x_{m}, y_{b}=y_{m}\right)$, $m=1, \ldots, 704$.

Thus, the membership value of the $m$-th rule can be calculated as

$$
\operatorname{Val}\left(R_{j}^{m}\right)=\exp \left(-\frac{\left(r_{j b}-s_{j m}\right)^{T}\left(r_{j b}-s_{j m}\right)}{2 \sigma^{2}}\right), j=1,2, \ldots, 6,
$$

where $\sigma$ is the smoothing parameter.

The inference engine is a combination of the product inference engine with the algebraic product for the $t$-norm operator. Thus, the fuzzy inference can be calculated as

$$
\operatorname{Val}\left(R^{m}\right)=\operatorname{Val}\left(R_{1}^{m}\right) \times \operatorname{Val}\left(R_{2}^{m}\right) \times \ldots \times \operatorname{Val}\left(R_{6}^{m}\right) .
$$

Lastly, the following center average defuzzifier is used to estimate the object coordinates: 


$$
\begin{gathered}
x_{b}=\frac{\sum_{m=1}^{N} x_{m} \times \operatorname{Val}\left(R^{m}\right)}{\sum_{m=1}^{N} \operatorname{Val}\left(R^{m}\right)}, \\
y_{b}=\frac{\sum_{m=1}^{N} y_{m} \times \operatorname{Val}\left(R^{m}\right)}{\sum_{m=1}^{N} \operatorname{Val}\left(R^{m}\right)} .
\end{gathered}
$$

\section{Experiments}

In our research, several experiments were implemented to demonstrate the superiority of the IPS we developed on the basis of the reference database. In our first experiment, two datasets include 288 points, and 392 points are sensed and collected to be the test blind nodes. For each test blind dataset, several positioning tests were performed under different conditions. In the first test, all antenna functions are assumed to be good. In the second test, one antenna is assumed to be nonfunctional. In the third and fourth tests, the two and three antennas are considered to be in the failed states. However, only the 10 closest neighbor points with the closest RSS signals between the reference and blind nodes are used for the positioning estimation in all the tests. ${ }^{(21)}$ Tables 2 and 3 show some of the estimated mean absolute errors (MAEs) for 288 and 392 test points, respectively. From the experimental results shown, the positioning performance of the developed IPS is clearly found to be still good even when some of the antennas fail.

Continuously, in the second experiment, different tests based on 440 sensed points are carried out. Here, only $\sigma=0.01$ is considered in all the tests. Table 4 shows the positioning MAEs. From the results shown in Table 4, the positioning error of the developed IPS is still accurate even when three or four antennas fail.

Table 2

Estimated MAEs (cm) for 288 test points.

\begin{tabular}{lcccc}
\hline$\sigma$ & No antenna fails & (1) fails & $(1),(6)$ fail & $(2),(4),(6)$ fail \\
\hline 0.01 & 4.1529 & 6.2430 & 11.5280 & 23.5040 \\
0.02 & 8.3464 & 13.6264 & 21.5208 & 37.6253 \\
\hline
\end{tabular}

Table 3

Estimated MAEs (cm) for 392 test points.

\begin{tabular}{lcccc}
\hline$\sigma$ & No antenna fails & (1) fails & $(1),(6)$ fail & $(2),(4),(6)$ fail \\
\hline 0.01 & 0.0835 & 0.2397 & 1.1725 & 6.8534 \\
0.02 & 2.4429 & 5.9614 & 12.6204 & 23.6365 \\
\hline
\end{tabular}


Table 4

Estimated MAEs (cm) for 440 test points for different conditions.

\begin{tabular}{lclc}
\hline Failed antenna & MAE $(\mathrm{cm})$ & Failed antenna & MAE $(\mathrm{cm})$ \\
\hline No one & 3.1418 & $(2),(6)$ & 12.8770 \\
$(1)$ & 4.5104 & $(3),(6)$ & 12.2751 \\
$(2)$ & 6.0525 & $(1),(3),(5)$ & 19.1460 \\
$(3)$ & 5.6997 & $(2),(4),(6)$ & 21.4786 \\
$(4)$ & 4.8890 & $(1),(2),(3),(4)$ & 38.7960 \\
$(5)$ & 6.2270 & $(3),(4),(5),(6)$ & 40.0225 \\
$(6)$ & 6.2172 & $(1),(2),(4),(5)$ & 37.1837 \\
$(1),(3)$ & 11.0516 & $(1),(2),(3),(4),(5)$ & 53.3390 \\
$(1),(5)$ & 9.3343 & $(2),(3),(4),(5),(6)$ & 54.5354 \\
$(2),(4)$ & 10.7836 & $(1),(2),(4),(5),(6)$ & 55.3309
\end{tabular}

\section{Conclusion}

In this research, an IPS composed of Sparklan antennas and a Zigbee module is constructed. The IP experiments under different conditions are performed. From the obtained experimental results, the positioning accuracy is clearly found to be still good even when some of the antennas are nonfunctional. This shows that the proposed IPS can be used in real applications. In addition, only 704 sensed points are used as reference nodes for IP application. We certainly believe that IP accuracy will be improved effectively if more reference nodes are created.

\section{Acknowledgments}

This research was partially supported by the Ministry of Science and Technology, Taiwan, under Contract No. MOST-107-2221-E-214-029.

\section{References}

1 Y. Y. Gu, A. Lo, and I. Niemegeers: IEEE Commun. Surv. Tutorials 11 (2009) 13. https://doi.org/10.1109/ SURV.2009.090103

2 H. Liu, H. Darabi, P. Banerjee, and J. Liu: IEEE Trans. Syst. Man Cybern. 37 (2007) 1067. https://doi. org/10.1109/TSMCC.2007.905750

3 G. W. Shi and Y. Ming: Lecture Notes Electr. Eng. Wireless Commun. Networking Appl. 348 (2016) 1269.

4 B. Kim, W. Bong, and Y. C. Kim: Proc. 2011 IEEE Consumer Communications and Networking Conf. (IEEE, 2011) 933-936. https://doi.org/10.1109/CCNC.2011.5766644

5 M. Vossiek, L. Wiebking, P. Gulden, J. Wiehardt, C. Hoffmann, and P. Heide: IEEE Microwave Mag. 4 (2003) 77. https://doi.org/10.1109/MMW.2003.1266069

6 G. Nuno-Barrau and J. M. Paez-Borrallo: EURASIP J. Appl. Signal Process. 1 (2006) 159. https://doi. org/10.1155/ASP/2006/68154

7 T. Roos, P. Myllymaki, H. Tirri, P. Misikangas, and J. Sievanan: Int. J. Wireless Inf. Networks 9 (2002) 155. https://doi.org/10.1023/A:1016003126882

8 V. Honkavirta, T. Perala, S. Ali-Loytty, and R. Piche: Proc. 6th Workshop on Positioning, Navigation and Communication (IEEE, 2009) 243-251. https://doi.org/10.1109/WPNC.2009.4907834

9 M. Y. Umair, K. V. Ramana, and D. K. Yang: Proc. 2014 IEEE Conf. Computers, Communications and Its Applications (IEEE, 2014) 19-23. https://doi.org/10.1109/ComComAp.2014.7017163

10 K. F. S. Wong, I. W. Tsang, V. Cheung, S. H. G. Chan, and S. J. T. Kwok: Proc. 2005 IEEE Global Telecommunications Conference (IEEE, 2005) 2772-2776. https://doi.org/10.1109/GLOCOM.2005.1578264

11 C. Savarese, J. M. Rabaey, and J. Beutel: Proc. 2001 IEEE Int. Conf. Acoustics, Speech, and Signal Processing (IEEE, 2001) 2037-2040. https://doi.org/10.1109/ICASSP.2001.940391 
12 H. D. Chon, S. Jun, H. Jung, and S. W. An: J. Global Positioning Syst. 3 (2004) 32. https://doi.org/10.5081/ jgps.3.1.32

13 H. L. Ding, W. W. Y. Ng, P. P. K. Chan, D. L. Wu, X. L. Chen, and D. S. Yeung: Proc. IEEE 2010 Int. Conf. Machine Learning and Cybernetics (IEEE, 2010) 1147-1152. https://doi.org/10.1109/ICMLC.2010.5580925

14 A. K. M. Mahtab Hossain and W. S. Soh: Proc. 18th Annual IEEE International Symposium on Personal, Indoor and Mobile Radio Communications (IEEE, 2007). https://doi.org/10.1109/PIMRC.2007.4394215

15 F. Subhan, H. Hasbullah, A. Rozyyev, and S. T. Bakhsh: Proc. 2011 Int. Conf. Information Science and Applications (2011). https://doi.org/10.1109/ICISA.2011.5772436

16 W. P. Chen and X. F. Meng: Proc. 14th IEEE Int. Conf. Networks (IEEE, 2006). https://doi.org/10.1109/ ICON.2006.302647

17 G. Goncalo and S. Helena: Proc. Third Int. Conf. Sensor Technologies and Applications (2009) 152-157. https://doi.org/10.1109/SENSORCOMM.2009.31

18 https://manualzz.com/doc/2766869/sparklan-ax-106m-network-antenna

19 J. Yen and R. Langari: Fuzzy Logic: Intelligence, Control, and Information (Prentice Hall Inc., Upper Saddle River, New Jersey 1999) p. 183.

20 C. Y. Chen, Y. J. Chen, S. W. Chen, C. Y. Shen, and R. C. Hwang: J. Electr. Electron. Eng. 4 (2016) 97. https:// doi.org/10.11648/j.jeee.20160405.12

21 C. Y. Chen, S. W. Chen, Y. J. Chen, and R. C. Hwang: Int. J. Future Comput. Commun. 7 (2018) 74. https://doi. org/10.18178/ijfcc.2018.7.3.524

\section{About the Authors}

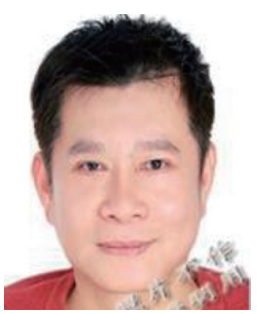

Huang-Chu Huang received his Ph.D. degree in electrical engineering from National Sun Yat-Sen University, Taiwan, in year 2001. Currently, he is a professor of the Electronic Communication Department, National Kaohsiung University of Science and Technology, Kaohsiung City, Taiwan. His research interests are in the areas of control, power signal prediction, and neural network applications. (h4530@nkust.edu.tw)

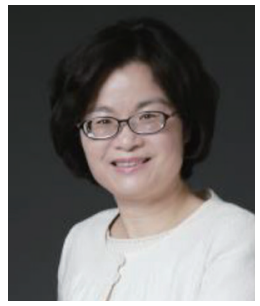

Chi-Yen Shen received her B.S. degree in electrical engineering from ChengKung University in Taiwan in 1987, her Ph.D. degree in electrical engineering from National Sun Yat-Sen University in Taiwan in 1991. She is currently a professor of Electrical Engineering at I-Shou University, Taiwan. Her research interests are in surface acoustic wave devices, sensors, and electronic ceramics. (cyshen@isu.edu.tw)

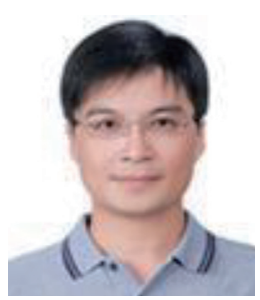

Chih-Yung Chen is an associate professor of the Department of Computer Science and Information Engineering at Shu-Te University, Taiwan. He received his Ph.D. degree in electrical engineering from I-Shou University in 2008. His current research interests are in artificial intelligence, autonomous robot control, database system, and indoor positioning technologies. (mikechen@stu.edu.tw ). 


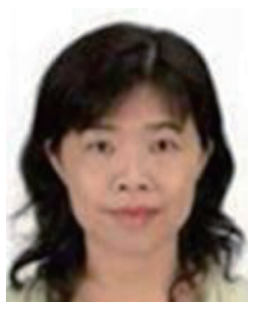

Yu-Ju Chen currently is an assistant professor of the Information Management Department at Cheng Shiu University. Her research interests include artificial intelligence, fuzzy theory, and information management.

(yjchen@csu.edu.tw)

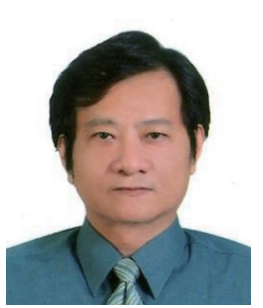

Rey-Chue Hwang received his Ph.D. degree in electrical engineering from Southern Methodist University, Dallas, TX, in 1993. Currently, he is a full professor of the Electrical Engineering Department, I-Shou University, Taiwan, R.O.C. Dr. Hwang has published more than 250 papers in various journals and conferences in the areas of artificial intelligent system, signal processing, and fuzzy control. He is now a Fellow of IET and a senior member of IEEE. He chartered the IEEE CIS Chapter, Tainan Section and served as the co-chair and chair from year 2004 to year 2009.

(rchwang@isu.edu.tw) 